\title{
Tratamiento nutricional hiperproteico precirugía bariátrica en obesidad mórbida
}

\author{
High-protein diet in morbidity obesity patient before bariatric surgery
}

\author{
Blanca R. Pardo-Pacheco ${ }^{1,2 *}$, Elizabeth Pérez-Cruz, Nayeli G. Nieto-Velázquez³, \\ Juan Asbun-Bojalit ${ }^{4}$ y Mario A. Moreno-Eutimio ${ }^{3}$ \\ ${ }^{1}$ Unidad de Soporte Nutricional y Metabolismo, Hospital Juárez de México; ${ }^{2}$ Escuela Superior de Enfermería y Obstetricia, Instituto Politécnico \\ Nacional; ${ }^{3}$ Unidad de Investigación en Inmunidad e Inflamación, Hospital Juárez de México; ${ }^{4}$ Departamento de Posgrado de la Facultad de Medicina, \\ Instituto Politécnico Nacional. Ciudad de México, México
}

\begin{abstract}
Resumen
Objetivo: Comparar la efectividad de un plan de alimentación hipocalórico hiperproteico con otro normoproteico sobre la composición corporal, los parámetros bioquímicos y las citocinas inflamatorias en pacientes obesos precirugía bariátrica sometidos a un tratamiento integral. Método: Se estudiaron 76 pacientes con un índice de masa corporal (IMC) $\geq 40 \mathrm{~kg} / \mathrm{m}^{2}$ previamente a la cirugía bariátrica. Un grupo fue tratado con una dieta hipocalórica hiperproteica y se comparó con una dieta hipocalórica normoproteica. Se evaluaron parámetros bioquímicos, parámetros antropométricos, composición corporal y valores de citocinas inflamatorias en suero al inicio y después de 4 meses de tratamiento. Resultados: En ambos grupos se observó una disminución de peso, de IMC y de masa grasa, así como un incremento de la masa muscular respecto al momento basal $(p<0.05)$, sin diferencias entre los grupos estudiados. No se encontraron cambios en los parámetros bioquímicos ni en las concentraciones séricas de factor de necrosis tumoral (TNF) e interleucina (IL)- 6 antes y después de 4 meses de tratamiento, ni entre los grupos evaluados $(p>0.05)$. Las concentraciones séricas de IL-1 $\beta$ disminuyeron únicamente con la dieta hipocalórica normoproteica $(p=0.02)$. Conclusiones: La dieta hipocalórica hiperproteica no muestra ventajas en la reducción de peso y grasa corporal, ni en la ganancia de masa muscular, en comparación con la dieta hipocalórica normoproteica en pacientes con obesidad mórbida precirugia bariátrica sometidos a un tratamiento integral.
\end{abstract}

PALABRAS CLAVE: Obesidad mórbida. Dieta hiperproteica. Cirugía bariátrica.

\section{Summary}

Objective: Compare the effectiveness of a hyperproteic hypocaloric feeding plan with a normoproteic on body composition, biochemical parameters and inflammatory cytokines in obese pre-bariatric surgery patients in the integral treatment. Method: Seventy-six pre-bariatric surgery patients with body mass index (BMI) $\geq 40 \mathrm{~kg} / \mathrm{m}^{2}$ were studied. One group was treated with a hyperproteic hypocaloric diet and compared with a normoproteic hypocaloric diet. Biochemical parameters, anthropometric parameters, body composition and levels of tumor necrosis factor (TNF), interleukin (IL)-6 and IL-1ß in serum were evaluated at the initiation of treatment and after 4 months. Results: In both groups studied, a decrease in weight, BMI and fat mass was observed, as well as an increase in muscle mass compared to baseline $(p<0.05)$, no differences showed between the groups studied. No change was found in the biochemical parameters and serum levels of TNF and IL-6 before and after 4 months of treatment, nor among the groups evaluated $(p>0.05)$. Serum IL-1 $\beta$ levels decreased after treatment with only a normoprotein hypocaloric diet $(p=0.02)$. Conclusions: Hyperproteic hypocaloric diet does not show advantages

\author{
Correspondencia: \\ *Blanca R. Pardo-Pacheco \\ Av. Instituto Politécnico Nacional, 5160 \\ Col. Magdalena de las Salinas, Del. Gustavo A. Madero Fecha de recepción: 23-03-2018 \\ C.P. 07760, Ciudad de México, México Fecha de aceptación: 14-05-2018 \\ E-mail: blaropp72@yahoo.com.mx \\ DOI: $10.24875 /$ CIRU.18000299
}

Cir Cir. 2018;86:508-514

Contents available at PubMed

www.cirugiaycirujanos.com 
in weight reduction and body fat or in muscle mass gain compared to the normoproteic hypocaloric diet in patients with morbid obesity bariatric pre-surgery in the integral treatment.

KEY WORDS: Morbid obesity. High-protein diet. Bariatric surgery.

\section{Introducción}

Actualmente la obesidad y el sobrepeso se definen como una acumulación anormal o excesiva de grasa que puede ser perjudicial para la salud, de etiología multifactorial y que se desarrolla a partir de la interacción de la influencia de factores genéticos, sociales, conductuales, psicológicos, metabólicos, celulares y moleculares'.

Según datos de la Organización Mundial de la Salud, la prevalencia de obesidad en el mundo se ha doblado entre 1980 y 2014. En 2016, más de 1900 millones de adultos a partir de 18 años de edad tenían sobrepeso, de los cuales más de 650 millones eran obesos; el $13 \%$ eran mujeres obesas ${ }^{2}$.En México, los resultados de la Encuesta Nacional en Salud y Nutrición 2016 reportan una prevalencia de sobrepeso y obesidad en adultos de 20 años o más del $72.5 \%$, con predomino del sexo femenino ${ }^{3}$.

El sobrepeso y la obesidad como resultado del desequilibrio entre energía y actividad física conlleva la hipertrofia y la hiperplasia del adipocito, sobrecargando su capacidad funcional, provocando angiogénesis e hipoxia del adipocito y reclutamiento de células del sistema inmunitario, como los macrófagos proinflamatorios que infiltran el adipocito ${ }^{4,5}$. Esta infiltración de macrófagos activa las vías de señalización que llevan a la producción de citocinas, como el factor de necrosis tumoral (TNF), la interleucina $1 \beta$ (IL-1 $\beta$ ) y la interleucina 6 (IL-6), que aunado a la proteína $C$ reactiva conducen a un estado de inflamación crónico de bajo grado e inhibición de los marcadores antiinflamatorios, así como a una disminución de la densidad de los receptores para la insulina y un aumento de los receptores $\beta 3$ que conduce a lipólisis. Estos efectos llevan a un mayor riesgo para el desarrollo de enfermedades cardiovasculares, diabetes tipo 2 y síndrome metabólico ${ }^{5,6}$.

Los cambios en el estilo de vida, regímenes dietéticos e incrementos en la actividad física pueden ser la primera línea de terapia en la disminución de la obesidad y sus complicaciones ${ }^{7}$. Diversos estudios demuestran que existen factores nutricionales que pueden modular el sistema inmunitario, entre los cuales se incluyen la ingesta calórica ${ }^{8}$, los tipos de grasas $^{9-11}$, los hidratos de carbono $0^{12,13}$ y la cantidad de proteínas $^{12}$, que repercuten de manera positiva sobre los marcadores inflamatorios, las modificaciones de la composición corporal y la reducción de los marcadores bioquímicos.

La intervención nutricional ha demostrado ser una intervención de bajo costo y alta efectividad en la reducción de peso ${ }^{7}$. Sin embargo, hasta el momento no hay datos suficientes que permitan generar recomendaciones sobre cuál es la mejor distribución de macronutrimentos, lo que nos daría una pauta para la implementación de protocolos de manejo dirigido al paciente con obesidad. El objetivo de este estudio fue evaluar la efectividad de un plan de alimentación hipocalórico hiperproteico sobre los cambios de composición corporal, el perfil de lípidos, la resistencia a la insulina y las citocinas inflamatorias en pacientes con obesidad mórbida previamente a la cirugía bariátrica, en comparación con un plan de alimentación hipocalórico normoproteico.

\section{Método}

\section{Población de estudio}

Se incluyeron hombres y mujeres mayores de 18 años y menores de 50 años con un índice de masa corporal $(I M C) \geq 40 \mathrm{~kg} / \mathrm{m}^{2}$, con o sin comorbilidad, que aceptaron participar en el estudio y firmaron la carta de consentimiento informado. No se incluyeron sujetos con enfermedades autoinmunitarias, enfermedad renal crónica, embarazadas o con tratamiento farmacológico de esteroides, antiinflamatorios no esteroideos o inmunomoduladores. El protocolo fue autorizado con el registro HJM 0121/16 por los Comités de Investigación, Ética en Investigación y Bioseguridad del Hospital Juárez de México.

\section{Diseño del estudio}

El grupo de estudio tuvo un plan de alimentación hipocalórico hiperproteico (grupo HP: hidratos de carbono $50 \%$, proteínas $25 \%$ y lípidos $25 \%$ ) y el grupo control tuvo un plan de alimentación hipocalórico normoproteico (grupo NP: hidratos de carbono $55 \%$, 
proteínas $15 \%$ y lípidos 30\%). Ambos grupos recibieron 500 calorías menos del consumo habitual, estimado por recordatorio de 24 horas, durante un período de 4 meses. Los dos grupos fueron tratados de manera integral, incluyendo tratamiento médico especializado, psicológico individual y familiar, nutricio y régimen de ejercicio.

La revisión médica especializada preoperatoria consistió en consulta con el médico internista y el médico endocrinólogo cada 2 meses, una consulta con el médico cardiovascular y una valoración preoperatoria por parte de cirugía bariátrica, y finalmente una evaluación de la función pulmonar mediante espirometría.

El manejo psicológico individual preoperatorio fue mediante consulta mensual, que consistió en un examen mental de inicio mediante entrevista estructurada, continuando con un cuestionario de ansiedad y depresión del inventario de depresión de Beck y seguido de una terapia centrada en soluciones cognitivo-conductuales. El manejo psicológico familiar preoperatorio y posoperatorio se realizó de forma mensual mediante terapia familiar sistémica, y se utilizó el índice de función familiar como instrumento de evaluación.

El apego al tratamiento nutricional fue evaluado mensualmente mediante el instrumento recordatorio de 24 horas y toma de mediciones antropométricas (peso) y de composición corporal (porcentaje de masa grasa y magra). Además, se realizó una intervención educativa que incluyó sesiones-taller cada 2 meses con actividades educativas, enfocadas a la modificación de los hábitos de alimentación.

El programa de ejercicio físico preoperatorio consistió en ejercicio físico aeróbico de cuatro a cinco veces por semana durante 30 minutos a intensidad moderada (60 a $70 \%$ de la frecuencia cardiaca máxi$\mathrm{ma})$, y se complementó con ejercicio de resistencia dos veces por semana con tres series de 10, trabajando diferentes músculos con el 5 al $10 \%$ del peso corporal.

\section{Determinaciones de parámetros bioquímicos, citocinas inflamatorias y composición corporal}

Se tomó una muestra de $5 \mathrm{ml}$ de sangre periférica al inicio y después de 4 meses de tratamiento para la determinación de los marcadores bioquímicos (glucosa en ayuno, insulina en ayuno, colesterol total, colesterol ligado a lipoproteínas de alta densidad [HDL] y a lipoproteínas de baja densidad [LDL]) mediante reacción enzimática colorimétrica con equipo automatizado (Analizador ADVIA 1800, Siemens), y la resistencia a la insulina mediante el modelo homeostático (HOMA).

Se tomó una muestra de $4 \mathrm{ml}$ de sangre periférica para determinar las concentraciones séricas de IL-6, IL-1 $\beta$ y TNF (en pg/ml) mediante el método de perlas de captura (Human Inflammatory Cytokine Kit, BD Cytometric Bead Array) para citometría de flujo (BD Accuri C6).

La composición corporal se determinó al inicio y al final de la intervención (porcentaje de masa grasa y porcentaje de masa magra) por bioimpedancia (Tanita MC-780). Al grupo con dieta hiperproteica se le realizó una prueba de función renal al inicio y al final del tratamiento nutricional.

\section{Análisis estadístico}

Se determinó la normalidad de los grupos mediante la prueba de Kolmogórov-Smirnov y se realizó una comparación de medias entre los grupos mediante la prueba t de Student para muestras independientes 0 la prueba de Mann Whitney. Se compararon las variables estudiadas antes y después del tratamiento nutricional en los grupos mediante $t$ de Student pareada o Wilcoxon. El análisis estadístico se realizó con el paquete estadístico SPSS versión 20.0, y se consideró diferencia estadísticamente significativa un valor de $p \leq 0.05$.

\section{Resultados}

De los 120 pacientes que llegaron a la Clínica de Obesidad y Trastornos Metabólicos durante el periodo de agosto de 2016 a agosto de 2017, 76 cumplieron los criterios de inclusión, participaron en el estudio y firmaron su carta de consentimiento. El grupo NP estuvo conformado por 39 pacientes, de los cuales el $77 \%$ eran mujeres $(n=30)$ y el $23 \%$ eran hombres $(n=9)$, con una edad media de $35.1 \pm 8.01$ años. El grupo HP estuvo conformado por 37 pacientes, de los cuales el $81.1 \%(n=30)$ eran mujeres y el $18.9 \%$ eran hombres $(n=7)$, con una edad media de $35.7 \pm 7.64$ años. Tres participantes se excluyeron por abandono del tratamiento por decisión personal, uno del grupo NP y dos del grupo HP; ningún paciente presentó efectos adversos derivados de la dieta.

No se encontraron diferencias significativas al inicio del tratamiento en cuanto a edad, peso, IMC, 
Tabla 1. Datos demográficos al inicio del tratamiento integral de la población de estudio

\begin{tabular}{lccc}
\hline & $\begin{array}{c}\text { Grupo NP } \\
(\mathbf{n}=\mathbf{3 9})\end{array}$ & $\begin{array}{c}\text { Grupo HP } \\
(\mathbf{n}=\mathbf{3 7})\end{array}$ & $\mathbf{p}$ \\
\hline Edad (años) & $35.15 \pm 8.01$ & $35.78 \pm 7.64$ & 0.7272 \\
Peso (kg) & $120.20 \pm 19.26$ & $120.17 \pm 22.36$ & 0.9964 \\
IMC (kg/m²) & $45.32 \pm 6.02$ & $46.14 \pm 6.96$ & 0.5834 \\
Grasa (\%) & $54.30 \pm 7.23$ & $55.07 \pm 8.42$ & 0.3695 \\
Músculo (\%) & $45.39 \pm 7.40$ & $44.14 \pm 8.07$ & 0.3581 \\
PA (mm/Hg) & $100.29 \pm 9.65$ & $98.39 \pm 11.81$ & 0.2625 \\
Glucosa (mg/dl) & $97 \pm 34.27$ & $109 \pm 59.17$ & 0.7287 \\
Colesterol HDL (mg/dl) & $38.13 \pm 8.18$ & $40.89 \pm 11.42$ & 0.2324 \\
Colesterol LDL (mg/dl) & $115.47 \pm 29.57$ & $115.53 \pm 26.89$ & 0.9916 \\
Colesterol total (mg/dl) & $163.28 \pm 31.06$ & $167.48 \pm 32.04$ & 0.5602 \\
Rl (HOMA) & $7.03 \pm 3.78$ & $6.77 \pm 5.45$ & 0.2428 \\
TNF (pg/ml) & $4.8 \pm 1.47$ & $9.3 \pm 3.41$ & 0.9089 \\
IL1- $\beta$ (pg/ml) & $10.5 \pm 2.49$ & $8.7 \pm 2.29$ & 0.6226 \\
IL-6 (pg/ml) & $3.7 \pm 0.65$ & $2.4 \pm 0.56$ & 0.8132 \\
Comorbilidad & $87.18 \%$ & $78.38 \%$ & $0 \%$ \\
1 & $53.84 \%$ & $37.83 \%$ & - \\
2 <3 & $23.07 \%$ & $32.43 \%$ & - \\
\hline & $10.25 \%$ & $8.10 \%$ & - \\
\hline
\end{tabular}

HDL: lipoproteínas de alta densidad; HP: régimen hipocalórico hiperproteico;

IL: interleucina; IMC: índice de masa corporal; LDL: lipoproteínas de baja densidad;

$\mathrm{NP}$ : régimen hipocalórico normoproteico; $\mathrm{PA}$ : presión arterial; RI: resistencia a la insulina;

TNF: factor de necrosis tumoral.

porcentaje de grasa y músculo, presión arterial, parámetros bioquímicos y citocinas séricas entre los grupos de estudio (Tabla 1).

\section{Peso y composición corporal}

El grupo NP tuvo un peso inicial de $120.2 \pm 19.26 \mathrm{~kg}$ $y$ un peso final de $115.21 \pm 18.99 \mathrm{~kg}(p<0.001)$, y un IMC inicial de $45.32 \pm 6.02 \mathrm{~kg} / \mathrm{m}^{2}$ y un IMC final de $43.48 \pm 6.12 \mathrm{~kg} / \mathrm{m}^{2}(\mathrm{p}<0.001)$, lo que representó una pérdida del $4.1 \%$ en promedio durante 4 meses de tratamiento. El porcentaje de masa grasa inicial fue del $54.30 \pm 7.23 \%$ y el final fue del $51.29 \pm 8.07 \%$ $(p=0.0005)$, y el porcentaje de masa muscular inicial fue del $45.39 \pm 7.40 \%$ y el final fue del $48.78 \pm 8.19 \%$ $(p=0.0002)$, lo que representó una disminución del $2.58 \%$ y un aumento del $2.95 \%$, respectivamente, después de 4 meses de tratamiento (Fig. 1).

El grupo HP inició con un peso promedio de 120.17 $\pm 22.36 \mathrm{~kg}$ y finalizó con $113.83 \pm 21.98 \mathrm{~kg}(p=0.001)$, y el IMC inicial fue de $46.14 \pm 6.96 \mathrm{~kg} / \mathrm{m}^{2}$ y el final de $43.70 \pm 6.94 \mathrm{~kg} / \mathrm{m}^{2}(p<0.001)$, lo que representa una
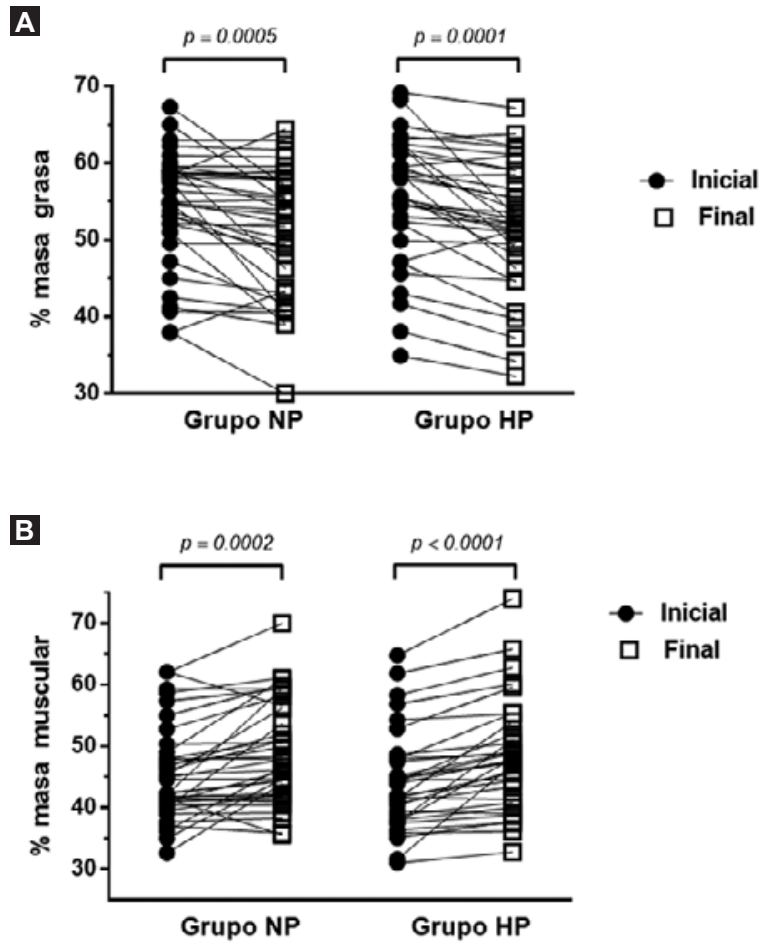

Figura 1. Efecto de las dietas hipocalóricas normoproteica e hiperproteica en sujetos con obesidad mórbida sometidos a un tratamiento integral. Porcentaje de masa grasa total (A) y porcentaje de masa muscular total (B) de pacientes con obesidad mórbida sometidos durante 16 semanas a un régimen hipocalórico normoproteico (grupo $N P, n=39$ ) o a un régimen hipocalórico hiperproteico (grupo $H P$, $n=37$ ). Prueba t de Student pareada antes y después del tratamiento; los valores de $p$ se muestran en la gráfica.

pérdida de peso del $5.27 \%$, sin diferencia estadística con el grupo NP $(p>0.05)$. El porcentaje de grasa inicial del grupo HP fue del $55.07 \pm 8.42 \%$ y el final fue del $51.82 \pm 8.37 \%(p=0.0001)$, y el porcentaje de masa muscular inicial fue del $44.14 \pm 8.07$ y el final de $48.29 \pm 8.93 \%(p=0.0001)$, lo que representó una disminución del $3.25 \%$ y un aumento del 3.74\%, respectivamente, después de 4 meses de tratamiento. No hubo diferencias significativas en la pérdida de grasa y la ganancia de masa muscular entre los grupos ( $p>0.05)$ (Fig. 1).

\section{Parámetros bioquímicos}

En el grupo NP, la glucosa en ayuno (inicial 97.34 \pm 34.27 y final $93.6 \pm 18.93 \mathrm{mg} / \mathrm{dl} ; \mathrm{p}=0.5179$ ), el colesterol HDL (inicial $38.13 \pm 8.18$ y final $38.30 \pm$ $8.35 \mathrm{mg} / \mathrm{dl} ; \mathrm{p}=0.5110$ ), el colesterol LDL (inicial $115.47 \pm 29.57$ y final $110.41 \pm 29.51 \mathrm{mg} / \mathrm{dl} ; p=0.2227$ ), el colesterol total (inicial $163.28 \pm 31.06$ y final 160.73 $\pm 33.00 \mathrm{mg} / \mathrm{dl} ; \mathrm{p}=0.4103$ ) y la Rl (inicial $7.03 \pm 3.78$ 
Tabla 2. Parámetros bioquímicos antes y después del tratamiento integral en los grupos de estudio

\begin{tabular}{|c|c|c|c|c|c|c|}
\hline & \multicolumn{3}{|c|}{ Grupo NP $(n=39)$} & \multicolumn{3}{|c|}{ Grupo HP $(n=37)$} \\
\hline & Inicio & Final & $\mathrm{p}$ & Inicio & Final & $\mathrm{p}$ \\
\hline Glucosa (mg/dl) & $97 \pm 34.27$ & $93.68 \pm 18.93$ & 0.5179 & $109 \pm 59.17$ & $96.43 \pm 22.80$ & 0.0584 \\
\hline $\mathrm{HDL}(\mathrm{mg} / \mathrm{dl})$ & $38.13 \pm 8.18$ & $38.38 \pm 8.35$ & 0.5110 & $40.89 \pm 11.42$ & $41.56 \pm 10.33$ & 0.3202 \\
\hline $\mathrm{LDL}(\mathrm{mg} / \mathrm{dl})$ & $115.47 \pm 29.57$ & $110.41 \pm 29.51$ & 0.2227 & $115.53 \pm 26.89$ & $109.30 \pm 27.57$ & 0.2435 \\
\hline Colesterol (mg/dl) & $163.28 \pm 31.06$ & $160.73 \pm 33.00$ & 0.4103 & $167.48 \pm 32.0$ & $164 \pm 27.65$ & 0.6554 \\
\hline $\mathrm{Rl}$ & $7.03 \pm 3.78$ & $6.50 \pm 3.66$ & 0.4231 & $6.77 \pm 5.45$ & $5.82 \pm 3.90$ & 0.1858 \\
\hline
\end{tabular}

HDL: lipoproteínas de alta densidad; HP: régimen hipocalórico hiperproteico; LDL: lipoproteínas de baja densidad; NP: régimen hipocalórico normoproteico; RI: resistencia a la insulina.

Tabla 3. Citocinas inflamatorias antes y después del tratamiento integral en los grupos de estudio

\begin{tabular}{|c|c|c|c|c|c|c|}
\hline & \multicolumn{3}{|c|}{ Grupo NP $(n=39)$} & \multicolumn{3}{|c|}{ Grupo HP $(n=37)$} \\
\hline & Inicio & Final & $\mathrm{p}$ & Inicio & Final & $\mathrm{p}$ \\
\hline TNF (pg/ml) & $4.8 \pm 1.47$ & $5.1 \pm 1.96$ & 0.8125 & $9.3 \pm 3.41$ & $4.8 \pm 1.40$ & 0.999 \\
\hline IL-1 $\beta(p g / m l)$ & $10.5 \pm 2.49$ & $7.8 \pm 2.05$ & 0.0234 & $8.7 \pm 2.29$ & $6.8 \pm 1.90$ & 0.3750 \\
\hline IL-6 (pg/ml) & $3.7 \pm 0.65$ & $3.6 \pm 0.70$ & 0.999 & $2.4 \pm 0.56$ & $1.6 \pm 0.48$ & 0.2031 \\
\hline
\end{tabular}

y final $6.50 \pm 3.66 ; p=0.4231$ ) no mostraron diferencias significativas con la dieta normoproteica después de 4 meses de tratamiento.

En el grupo HP, la glucosa en ayuno (inicial $109 \pm$ 59.17 y final $96.43 \pm 22.80 \mathrm{mg} / \mathrm{dl} ; \mathrm{p}=0.0584$ ), el colesterol HDL (inicial $40.89 \pm 11.42$ y final $41.56 \pm$ $10.33 \mathrm{mg} / \mathrm{dl} ; \mathrm{p}=0.3202$ ), el colesterol LDL (inicial $115.53 \pm 26.89$ y final $109.30 \pm 27.57 \mathrm{mg} / \mathrm{dl} ; p=0.2435$ ), el colesterol total (inicial $167.48 \pm 32.04$ y final 164.40 $\pm 27.65 \mathrm{mg} / \mathrm{dl} ; \mathrm{p}=0.6554)$ y la Rl $(6.77 \pm 5.45 \mathrm{y}$ final $5.82 \pm 3.90 ; p=0.1858)$ no mostraron diferencias significativas después del tratamiento. Los parámetros bioquímicos no mostraron diferencias entre los grupos NP y HP ( $p>0.05$ ) (Tabla 2$)$.

\section{Concentraciones séricas de citocinas inflamatorias}

En el grupo NP, las concentraciones séricas de IL-1 $\beta$ disminuyeron en un $25 \%$ después del tratamiento (inicial $10.5 \pm 2.49$ y final $7.8 \pm 2.05 \mathrm{pg} / \mathrm{ml} ; \mathrm{p}=0.0234$ ), mientras que el TNF (inicial $4.8 \pm 1.47$ y final $5.1 \pm$ $1.96 \mathrm{pg} / \mathrm{ml} ; \mathrm{p}=0.8125$ ) y la IL-6 (inicial $3.7 \pm 0.65 \mathrm{y}$ final $3.6 \pm 0.70 \mathrm{pg} / \mathrm{ml} ; p=0.9999$ ) no mostraron cambios estadísticamente significativos después de 4 meses de tratamiento. En el grupo HP, las concentraciones séricas de IL-1 $\beta$ (inicial $0.87 \pm 2.29$ y final $0.68 \pm$ $1.90 \mathrm{pg} / \mathrm{ml} ; p=0.3750$ ), TNF (inicial $0.93 \pm 3.41$ y final $0.48 \pm 1.40 \mathrm{pg} / \mathrm{ml} ; p=0.9999$ ) e IL-6 (inicial $0.24 \pm 0.56$ $y$ final $0.16 \pm 0 \mathrm{pg} / \mathrm{ml} ; \mathrm{p}=0.2031$ ) no tuvieron cambios significativos después del tratamiento con la dieta hiperproteica. No hubo cambios significativos entre los grupos (Tabla 3).

\section{Discusión}

Las dietas hipocalóricas con reducción leve a moderada de calorías tienen un impacto en la reducción de peso en pacientes con obesidad. Diversos estudios $^{14-17}$ han reportado una reducción de peso entre el 5 y el 10\%, con disminución de la grasa corporal y con un ligero aumento o conservación de la masa muscular y mejora en los parámetros bioquímicos en pacientes con sobrepeso u obesidad. Nosotros encontramos una reducción del 4.1 y el $5.2 \%$ en el peso en 4 meses de tratamiento en pacientes con obesidad mórbida, lo cual concuerda con lo reportado ${ }^{14-17}$.

Sacks, et al. ${ }^{14}$ evaluaron cuatro tipos de dietas hipocalóricas, dos de ellas hiperproteicas, en pacientes con sobrepeso u obesidad (IMC de 25 a $40 \mathrm{~kg} / \mathrm{m}^{2}$ ) y sin comorbilidad, durante 6 meses, y encontraron una reducción de peso similar en todos los grupos evaluados, con disminución en el perfil de lípidos y mejor sensibilidad a la insulina independientemente de la distribución de los macronutrientes. Nuestro estudio también encontró reducción de peso independiente de la distribución de las proteínas en la dieta, sin encontrar mejora en el perfil de lípidos y la resistencia 
a la insulina; estas diferencias podrían deberse al grado de obesidad (obesidad mórbida) y a la mayor resistencia a la insulina de nuestros pacientes con respecto a la población estudiada por Sacks, et al. ${ }^{14}$, además de a la presencia de comorbilidad y el menor tiempo de intervención (4 meses).

Salas-Salvado, et al..$^{15}$ estudiaron una población similar a la de nuestro estudio (obesos mórbidos), pero sin comorbilidad, con una dieta hipocalórica estricta (800 calorías totales) e hiperproteica con un $10 \%$ más de contenido proteico, y encontraron una reducción de los parámetros bioquímicos y de las citocinas inflamatorias; nuestro estudio no halló diferencias en estos parámetros, probablemente por las diferentes condiciones del estudio y la heterogeneidad de las características socioeconómicas, culturales y geográficas.

En relación con los marcadores inflamatorios, en nuestro estudio los pacientes mostraron una tendencia a la disminución, que fue significativa para las concentraciones séricas de IL-1 $\beta$ de los pacientes del grupo NP. Se conoce que el TNF, la IL-1 $\beta$ y la IL-6 se sobreexpresan localmente en tejido adiposo, pudiendo llegar a un aumento sistémico, por lo que nosotros evaluamos las citocinas sistémicamente, como reflejo de lo que pudiera estar ocurriendo en el tejido adiposo; sin embargo, se detectaron valores muy bajos de citocinas, muy cercanos al nivel mínimo detectado por el método utilizado, lo que podría no estar reflejando lo que ocurre en el tejido graso de los sujetos con obesidad mórbida. Por otra parte, las concentraciones séricas de citocinas están influenciadas por otras afecciones, como diabetes, hiperuricemias y dislipidemias, entre otras. Además, siempre que existan mediadores proinflamatorios se activan mecanismos de regulación, como la liberación de IL-10 y Factor de Crecimiento Transformante (TGF)- $\beta$ que tienen propiedades antiinflamatorias y la liberación del receptor soluble del TNF y el receptor antagónico de la IL-1 $\beta$, que neutralizan los efectos de sus respectivas citocinas ${ }^{18}$.

Aunque los estudios de Salas-Salvado, et al..$^{15} \mathrm{y}$ Bruun, et al..$^{19}$ mostraron diferencias en las concentraciones séricas de citocinas, esta aparente discrepancia con nuestro estudio pudiera explicarse por la presencia de comorbilidad, el grado de obesidad y el estricto régimen de tratamiento de los pacientes estudiados. Serán necesarios futuros estudios para evaluar nuestro tratamiento, con un mayor tiempo de intervención por tratarse de obesos mórbidos.

Nuestros hallazgos se suman a los resultados encontrados por otros autores: el paciente obeso, al continuar con esta condición, sigue presentando los inductores para disparar una inflamación crónica de bajo grado, y esto es más marcado en los obesos mórbidos por presentar una mayor cantidad de masa grasa. Finalmente, la adherencia al plan dietético es el factor más decisivo para lograr una pérdida ponderal significativa. Por tanto, estas dietas tienen la ventaja de adaptarse de manera individual respetando preferencias personales, hábitos, cultura y disponibilidad económica, y con mayor posibilidad de éxito a largo plazo.

\section{Conclusión}

La dieta hipocalórica hiperproteica no muestra ventajas en la reducción de peso y de grasa corporal ni en la ganancia de masa muscular en comparación con la dieta hipocalórica normoproteica en pacientes con obesidad mórbida sometidos a un tratamiento integral. La dieta hipocalórica en pacientes con obesidad mórbida, independientemente de su distribución de macronutrimentos, logra una reducción de peso y mejora la composición corporal.

\section{Conflicto de intereses}

Los autores declaran no tener conflictos de intereses.

\section{Bibliografía}

1. Obesity: preventing and managing the global epidemic. Report of a WHO consultation. World Health Organ Tech Rep Ser. 2000;894:i-xii.1-253.

2. Organización Mundial de la Salud. Ginebra: OMS; 2017. (Actualizado en octubre de 2017; consultado el 9 de febrero de 2018.) Obesidad y sobrepeso: nota descriptiva. Disponible en: http://www.who.int/mediacentre/factsheets/fs311/es

3. Encuesta Nacional de Salud y Nutrición de Medio Camino 2016 (ENSANUT 2016). Informe final de resultados. Instituto Nacional de Salud Pública. 31 de octubre de 2016. (Consultado el 9 de febrero de 2018.) Disponible en: http://transparencia.insp.mx/2017/auditorias-insp/12701_ Resultados_Encuesta_ENSANUT_MC2016.pdf

4. Zeyda M, Stulnig TM. Adipose tissue macrophages. Immunol Lett. 2007;112:61-7.

5. Dandona P, Aljada A, Bandyopadhyay A. Inflammation: the link between insulin resistance, obesity and diabetes. Trends Immunol. 2004;25:4-7.

6. Gregor MF, Hotamisligil GS. Inflammatory mechanisms in obesity. Annu Rev Immunol. 2011;29:415-45.

7. Yumuk V, Tsigos C, Fried M, Schindler K, Busetto L, Micic D, et al. European guidelines for obesity management in adults. Obes Facts. 2015;8:402-24.

8. Reilly SM, Saltiel AR. Adapting to obesity with adipose tissue inflammation. Nat Rev Endocrinol. 2017;13:633-43.

9. Siri-Tarino PW, Sun Q, Hu FB, Krauss RM. Meta-analysis of prospective cohort studies evaluating the association of saturated fat with cardiovascular disease. Am J Clin Nutr. 2010;91:535-46.

10. Cruz-Teno C, Pérez-Martínez $P$, Delgado-Lista J, Yubero-Serrano EM, García-Ríos A, Marín C, et al. Dietary fat modifies the postprandial inflammatory state in subjects with metabolic syndrome: the LIPGENE study. Mol Nutr Food Res. 2012;56:854-65.

11. Bonfanti N, Fernández JM, Gómez-Delgado F, Pérez-Jiménez F. [Effect of two hypocaloric diets and their combination with physical exercise on basal metabolic rate and body composition]. Nutr Hosp. 2014;29:635-43. 
12. Merino J, Kones R, Ferré R, Plana N, Girona J, Aragonés G, et al. Negative effect of a low-carbohydrate, high-protein, high-fat diet on small peripheral artery reactivity in patients with increased cardiovascular risk. Br J Nutr. 2013;109:1241-7.

13. Volek JS, Ballard KD, Silvestre R, Judelson DA, Quann EE, Forsythe CE, et al. Effects of dietary carbohydrate restriction versus low-fat diet on flow-mediated dilation. Metabolism. 2009;58:1769-77.

14. Sacks FM, Bray GA, Carey VJ, Smith SR, Ryan DH, Anton SD, et al. Comparison of weight-loss diets with different compositions of fat, protein, and carbohydrates. N Engl J Med. 2009;360:859-73.

15. Salas-Salvadó J, Bulló M, García-Lorda P, Figueredo R, Del Castillo D, Bonada A, et al. Subcutaneous adipose tissue cytokine production is not responsible for the restoration of systemic inflammation markers during weight loss. Int J Obes (Lond). 2006;30:1714-20.
16. Krieger JW, Sitren HS, Daniels MJ, Langkamp-Henken B. Effects of variation in protein and carbohydrate intake on body mass and composition during energy restriction: a meta-regression 1. Am J Clin Nutr. 2006;83:260-74.

17. García-Unciti M, Martínez JA, Izquierdo M, Gorostiaga EM, Grijalba A Ibáñez J. Effect of resistance training and hypocaloric diets with different protein content on body composition and lipid profile in hypercholesteroemic obese women. Nutr Hosp. 2012;27:1511-20.

18. Moreno-Eutimio MA, Acosta-Altamirano G. The immunometabolism of exercise and sedentary lifestyle. Cir Cir. 2014;82:344-51.

19. Bruun JM, Verdich C, Toubro S, Astrup A, Richelsen B. Association between measures of insulin sensitivity and circulating levels of interleukin-8, interleukin- 6 and tumor necrosis factor-alpha. Effect of weight loss in obese men. Eur J Endocrinol. 2003;148:535-42. 